\title{
Soluble PD-L1 is a potential biomarker of cutaneous melanoma aggressiveness and metastasis in obstructive sleep apnoea patients
}

\author{
Carolina Cubillos-Zapata ${ }^{1,2}$, Miguel Ángel Martínez-García ${ }^{3}$, \\ Francisco Campos-Rodríguez ${ }^{4}$, Manuel Sánchez de la Torre ${ }^{2,5}$, \\ Eduardo Nagore ${ }^{6}$, Antonio Martorell-Calatayud ${ }^{7}$, Luis Hernández Blasco 8,9 , \\ Eusebi Chiner Vives ${ }^{10}$, Jorge Abad-Capa ${ }^{2,11}$, Josep María Montserrat ${ }^{2,12}$, \\ Valentín Cabriada-Nuño (10 ${ }^{13}$, Irene Cano-Pumarega (10 ${ }^{14}$, \\ Jaime Corral-Peñafiel ${ }^{2,15}$, Trinidad Diaz-Cambriles ${ }^{16}$, Olga Mediano ${ }^{2,17}$ \\ María Somoza-González ${ }^{18}$, Joan Dalmau-Arias ${ }^{19}$, Isaac Almendros (102,20,21, \\ Ramón Farré2,20,21, Eduardo López-Collazo ${ }^{2,22,23}$, David Gozal ${ }^{24}$ and \\ Francisco García-Río ${ }^{1,2,25}$ on behalf of the Spanish Sleep Network
}

@ERSpublications

In patients with cutaneous melanoma, severe OSA increases serum levels of sPD-L1, which are associated with several indices of tumour aggressiveness and are an independent risk factor of sentinel lymph node metastasis http://ow.ly/qAU030mC7xx

Cite this article as: Cubillos-Zapata C, Martínez-García MÁ, Campos-Rodríguez F, et al. Soluble PD-L1 is a potential biomarker of cutaneous melanoma aggressiveness and metastasis in obstructive sleep apnoea patients. Eur Respir J 2019; 53: 1801298 [https://doi.org/10.1183/13993003.01298-2018].

ABSTRACT Obstructive sleep apnoea (OSA) upregulates the programmed cell death-1 receptor and its ligand (PD-L1) pathway, potentially compromising immunosurveillance. We compared circulating levels of soluble PD-L1 (sPD-L1) in patients with cutaneous melanoma according to the presence and severity of OSA, and evaluated relationships with tumour aggressiveness and invasiveness.

In a multicentre observational study, 360 patients with cutaneous melanoma underwent sleep studies, and serum sPD-L1 levels were assayed using ELISA. Cutaneous melanoma aggressiveness indices included mitotic rate, Breslow index, tumour ulceration, Clark level and tumour stage, and sentinel lymph node (SLN) metastasis was recorded as a marker of invasiveness.

sPD-L1 levels were higher in severe OSA compared to mild OSA or non-OSA patients. In OSA patients, sPD-L1 levels correlated with Breslow index and were higher in patients with tumour ulceration, advanced primary tumour stages or with locoregional disease. The incorporation of sPD-L1 to the classic risk factors to SLN metastasis led to net improvements in the classification of $27.3 \%$.

Thus, sPD-L1 levels are increased in melanoma patients with severe OSA, and, in addition, might serve as a potential biomarker of cutaneous melanoma aggressiveness and invasiveness in this group of subjects. 


\section{Introduction}

Obstructive sleep apnoea (OSA) is a highly prevalent disorder affecting $10-50 \%$ of middle-aged subjects [1], characterised by recurrent episodes of partial or complete upper airway obstruction associated with intermittent hypoxia and sleep fragmentation. These alterations induce oxidative stress, sympathetic activation and metabolic deregulation [2] and are associated with an increased risk of cardiovascular and neurocognitive consequences [3, 4]. More recently, significant associations between OSA and cancer prevalence, incidence and mortality have emerged [5-8], and more specifically, a relationship has been demonstrated between OSA and the aggressiveness of cutaneous melanoma $[8,9]$, the most lethal form of skin cancer, with a high tendency to metastasise rapidly [10].

A number of pathogenic mechanisms have been suggested to justify the possible correlation between OSA and cancer, ranging from the effects of oxidative stress on genetic malignant cell stability to the promotion of tumour angiogenesis [11, 12]. Furthermore, evidence suggesting alterations in immune function induced by intermittent hypoxia and sleep fragmentation have become apparent, including deregulation of tumour-associated macrophages and cytotoxic T-cell lymphocyte activity [13-16], which could compromise immunosurveillance and favour both the viability and progression of tumour cells. In this contextual setting, we have recently described intermittent hypoxia-induced increases in the expression of the pathway of programmed cell death (PD)-1 receptor and its ligand (PD-L1) among cancer-free OSA patients, and such changes would be anticipated to reduce autologous T-cell proliferation and cytotoxic activity of $\mathrm{CD}^{+}$lymphocytes, as well as increases in the recruitment of myeloid-derived suppressor cells [17], thereby providing biological plausibility to the increased incidence and aggressiveness of cancer as reported in these patients.

Of note, malignant tumour cells can also activate the PD-1/PD-L1 pathway to evade antitumoral immunity and promote malignant cell growth [18]. Recently, the soluble (s) form of PD-L1 has been detected in the blood of patients with tumours [19], and elevated sPD-L1 levels have been associated with worse prognosis in certain types of cancer [20]. For these reasons, sPD-L1 could provide a potential diagnostic, therapeutic or prognostic biomarker in patients with malignant tumours [21]. However, no information is available on whether the presence of OSA increases sPD-L1 levels in patients with cancer, or whether such levels are related to adverse tumour evolution in apnoeic subjects.

In the present study, we hypothesised that serum levels of sPD-L1 in patients with melanoma would be affected and enhanced in a severity-dependent fashion by the concurrent presence and severity of OSA. In addition, we evaluated the correlation of sPD-L1 levels with the aggressiveness and invasiveness of cutaneous melanoma, as inferred from a variety of well-established staging approaches as well as by the presence of sentinel lymph node (SLN) metastasis, the latter being considered as the most important factor in terms of patient survival [22].

\section{Methods}

Study subjects

In a multicentre, observational cross-sectional study performed in 29 teaching hospitals in Spain, newly diagnosed cutaneous melanoma patients aged $>18$ years were consecutively recruited from the dermatology

Affiliations: 'rupo de Enfermedades Respiratorias, Servicio de Neumología, Hospital Universitario La PazIdiPAZ, Madrid, Spain. ${ }^{2}$ Centro de Investigación Biomédica en Red en Enfermedades Respiratorias (CIBERES), Madrid, Spain. ${ }^{3}$ Respiratory Dept, Hospital Universitario y Politécnico la Fe, Valencia, Spain. ${ }^{4}$ Respiratory Dept, Hospital Universitario de Valme, Seville, Spain. ${ }^{5}$ Respiratory Dept, Group of Translational Research in Respiratory Medicine, Hospital Universitari Arnau de Vilanova and Santa Maria, IRBLleida, Lleida, Spain. ${ }^{6}$ Dermatology Dept, Instituto Valenciano de Oncología, Valencia, Spain. ${ }^{7}$ Dermatology Dept, Hospital de Manises, Valencia, Spain. ${ }^{8}$ Respiratory Dept, ISABIAL, Hospital General Universitario de Alicante, Alicante, Spain. ${ }^{9}$ Departamento Medicina Clinica, Universidad Miguel Hernandez, Elche, Spain. ${ }^{10}$ Respiratory Dept, Hospital san Juan de Alicante, Alicante, Spain. ${ }^{11}$ Respiratory Dept, Hospital Germans Trias i Pujol, Centro de investigacion Biomedica, Madrid, Spain. ${ }^{12}$ Respiratory Dept, Hospital Clinic (IDIBAPS), Barcelona, Spain. ${ }^{13}$ Respiratory Dept, Hospital Universitario Cruces, Bilbao, Spain. ${ }^{14}$ Respiratory Dept, Hospital Universitario de Getafe, Madrid, Spain. ${ }^{15}$ Respiratory Dept, Hospital Universitario S. Pedro Alcántara, Cáceres, Spain. ${ }^{16}$ Respiratory Dept, Hospital 12 de Octubre, Madrid, Spain. ${ }^{17}$ Respiratory Dept, Hospital Universitario de Guadalajara, Guadalajara, Spain. ${ }^{18}$ Pneumology Dept, Hospital Consorcio Terrassa, Barcelona, Spain. ${ }^{19}$ Dermatology Dept, Hospital de la Santa Creu i Sant Pau, Barcelona, Spain. ${ }^{20}$ Unitat de Biofísica i Bioenginyeria, Facultat de Medicina i Ciències de la Salut, Universitat de Barcelona, Barcelona, Spain.

${ }^{21}$ Institut d'Investigacions Biomèdiques August Pi i Sunyer (IDIBAPS), Barcelona, Spain. ${ }^{22}$ Tumorlmmunology Laboratory IdiPAZ, Madrid, Spain. ${ }^{23}$ Innate Immune Response Group, IdiPAZ, Madrid, Spain. ${ }^{24}$ Dept of Child Health, University of Missouri School of Medicine, Columbia, MO, USA. ${ }^{25}$ Facultad de Medicina, Universidad Autónoma de Madrid, Madrid, Spain.

Correspondence: Francisco García-Río, Hospital Universitario La Paz, IdiPAZ, Paseo de La Castellana 261, 28046 Madrid, Spain. E-mail: fgr01magmail.com 
or oncology units of each participating medical centre $[8,23]$. Exclusion criteria included extracutaneous location of melanoma, daytime respiratory or heart failure and current or previous use of home oxygen therapy, continuous positive airway pressure or noninvasive mechanical ventilation. The study was approved by the local ethics committees, and all participants signed informed consent.

\section{Dermatological evaluation}

As previously described [24], patients were evaluated by a dermatologist at each hospital, and recordings of tumour location (head or neck, upper extremities, trunk, lower extremities or acral) and clinical stage at diagnosis (categorised as localised (I-II) or locoregional disease or distant metastasis (III-IV)) were performed. All tumours were removed surgically and the following pathological features were determined. Breslow tumour thickness (in $\mathrm{mm}$ and categorised as $<1,1-2,2-4$ and $>4$ ), ulceration (presence or absence), tumour mitotic rate (mitoses $\cdot \mathrm{mm}^{-2}$ ) and Clark levels (level 1 or melanoma confined to the epidermis; level 2 or invasion into the papillary dermis; level 3 or invasion to the junction of the papillary and reticular dermis; level 4 or invasion into the reticular dermis; and level 5 or invasion into the subcutaneous fat) [25]. Based on tumour thickness, ulceration and mitotic rate, primary tumour categories were established, according to the American Joint Committee on Cancer (AJCC) 8th edition melanoma staging system [25]. In addition, SLN metastasis was recorded as a marker of cutaneous melanoma invasiveness and indicator of poor prognosis [22]. Classic predictors for SLN status included Breslow index (in $\mathrm{mm}$ ), presence of ulceration, mitotic index and Clark levels [26]. All dermatological and pathological evaluations were blinded to the sleep study and biochemical analysis findings.

\section{Sleep study}

All patients underwent overnight respiratory polygraphy within a maximum of 6 months after cutaneous melanoma diagnosis at the sleep unit of each hospital. The validated portable devices provided continuous recording of oronasal flow and pressure, thoracic and abdominal respiratory movements, heart rate and arterial oxygen saturation $\left(\mathrm{SaO}_{2}\right)$. All scoring and readings were conducted manually by experienced and trained personnel. Apnoea was defined as an interruption of oronasal flow of $>10 \mathrm{~s}$. Hypopnoea was defined as a $30-90 \%$ reduction in the oronasal airflow for $>10 \mathrm{~s}$ associated with an oxygen desaturation $\geqslant 3 \%$. The apnoea-hypopnoea index (AHI) was defined as the number of apnoeas plus hypopnoeas per hour of recording. In addition, the percentage of recording time with $\mathrm{SaO}_{2}<90 \%$, mean saturation, minimum saturation and oxygen desaturation index (ODI) were measured. According to the AHI, patients were divided into four groups: non-OSA $\left(<5\right.$ events $\left.\cdot \mathrm{h}^{-1}\right)$, mild OSA (5-14.9 events $\left.\cdot \mathrm{h}^{-1}\right)$, moderate OSA (15-29.9 events $\left.\cdot \mathrm{h}^{-1}\right)$ and severe OSA (>30 events $\left.\cdot \mathrm{h}^{-1}\right)$ [27].

\section{Determination of serum levels of soluble PD-L1}

Fasting venous blood samples were drawn between 08:00 h and 09:00 h. The blood samples were centrifuged to separate serum, and all specimens were immediately aliquoted, frozen and stored at $-80^{\circ} \mathrm{C}$. Concomitantly, serum sPD-L1 was assayed using the human PD-L1 enzyme-linked immunosorbent assay (ab214565; Abcam, Cambridge, UK) following the manufacturer's instructions for all samples. Measurements for serum samples were performed in duplicate. The detection limit of the assays was $2.91 \mathrm{pg} \cdot \mathrm{mL}^{-1}$, while intra-assay and inter-assay variations were $<20 \%$.

\section{Statistical analysis}

Continuous variables are expressed as mean \pm SD or median (interquartile range (IQR)), depending on their distribution, while categorical variables are reported as absolute numbers and percentages. Normality in the distribution of the data for each variable was explored using Shapiro-Wilk and skewness-kurtosis tests. Differences between groups were analysed using the Chi-squared test or Fisher's exact test (categorical variables) and Kruskal-Wallis test (ordinary or non-normal metric variables).

Potential associations between anthropometric and sleep parameters with sPD-L1 levels were evaluated using Spearman's correlation. Those variables exhibiting statistically significant findings were then introduced into a multiple linear regression analysis to identify independent determinants of sPD-L1 levels. Stepwise methods were used to include or remove individual independent variables at each step, based on the probability of F (entry 0.05 , removal 0.10 ). Other aspects explored included residual standard deviation, changes in the distribution of the residuals and the homogeneity of the variance over the predictors.

Associations between anthropometric and sleep parameters, classic risk factors and sPD-L1 levels with SLN metastasis were analysed by bivariate and forward stepwise multiple logistic regression. To assess whether the addition of sPD-L1 level to the classical aggressiveness markers improved the predictive power for invasion of SLN, we calculated the area under the receiver operating characteristics curve (AUC-ROC) for the classical model, with and without inclusion of sPD-L1 serum concentration. The equality of AUCs was assessed using 
the DeLong et al. [28] method. Net reclassification index (NRI) and integrated discrimination improvement (IDI) were applied to quantify the improvement contributed by this approach [29].

All tests were two-tailed, and a statistical significance level of 0.05 was retained. Analyses were performed using the Statistical Package for the Social Sciences (version 13.0; SPSS, Chicago, IL, USA) and MedCalc (www.medcalc.org).

\section{Results}

Out of 476 eligible patients with a diagnosis of cutaneous melanoma, 441 patients provided informed consent and were prospectively recruited. However, stored serum samples were not available for 81 patients, such that the remaining 360 patients were included in the current analysis (figure 1). Globally, 172 patients were female, mean \pm SD age was $57 \pm 15$ years, and mean body mass index (BMI) was $27.4 \pm 4.6 \mathrm{~kg} \cdot \mathrm{m}^{-2} .317$ patients $(88.1 \%)$ had local melanoma extension, and evidence of ulceration was found in 63 (17.5\%). The median (IQR) Breslow index was $0.81(0.49-1.80) \mathrm{mm}$, and the median (IQR) mitotic index was $1(0-2)$ cells $\cdot \mathrm{mm}^{-2}$. The overall prevalence rates of mild (AHI $<5$ events $\mathrm{h}^{-1}$ ) and moderate-to-severe (AHI $>15$ events. $\mathrm{h}^{-1}$ ) OSA were $30.3 \%$ and $33.1 \%$, respectively. The demographic and clinical characteristics of the four study groups are shown in table 1, and illustrates that as the severity of OSA increases, patients were increasingly more likely to be male, older and more overweight or obese.

SPD-L1 serum levels are elevated in cutaneous melanoma patients with severe OSA

sPD-L1 serum levels showed significant differences among the study groups $(p=0.035)$. When adjusted for sex, age, BMI and neck circumference, sPD-L1 levels were higher in severe OSA patients versus mild OSA or nonapnoeic patients (figure 2).

In the overall analysis of the cohort, sPD-L1 levels exhibited direct proportional relationships with AHI, ODI and level of nocturnal hypoxaemia, as well as with age, BMI and neck circumference (table 2). However, upon introduction of all the variables that reached significance into a multivariate linear regression analysis, only AHI was retained as an independent variable, both in for the whole cohort ( $\beta$ coefficient $0.983,95 \%$ CI $0.509-1.457 ; \mathrm{p}<0.001)$ and for patients with OSA ( $\beta$ coefficient $1.057,95 \%$ CI $0.512-1.638 ; \mathrm{p}<0.001$ ).

\section{SPD-L1 levels and melanoma aggressiveness in OSA patients}

Serum levels of SPD-L1 were higher in OSA patients with locoregional disease or metastasis than in patients with localised cutaneous melanoma $\left(96.6(58.6-200.0) \mathrm{pg} \cdot \mathrm{mL}^{-1}\right.$ versus $71.5(41.9-98.6) \mathrm{pg} \cdot \mathrm{mL}^{-1}$;

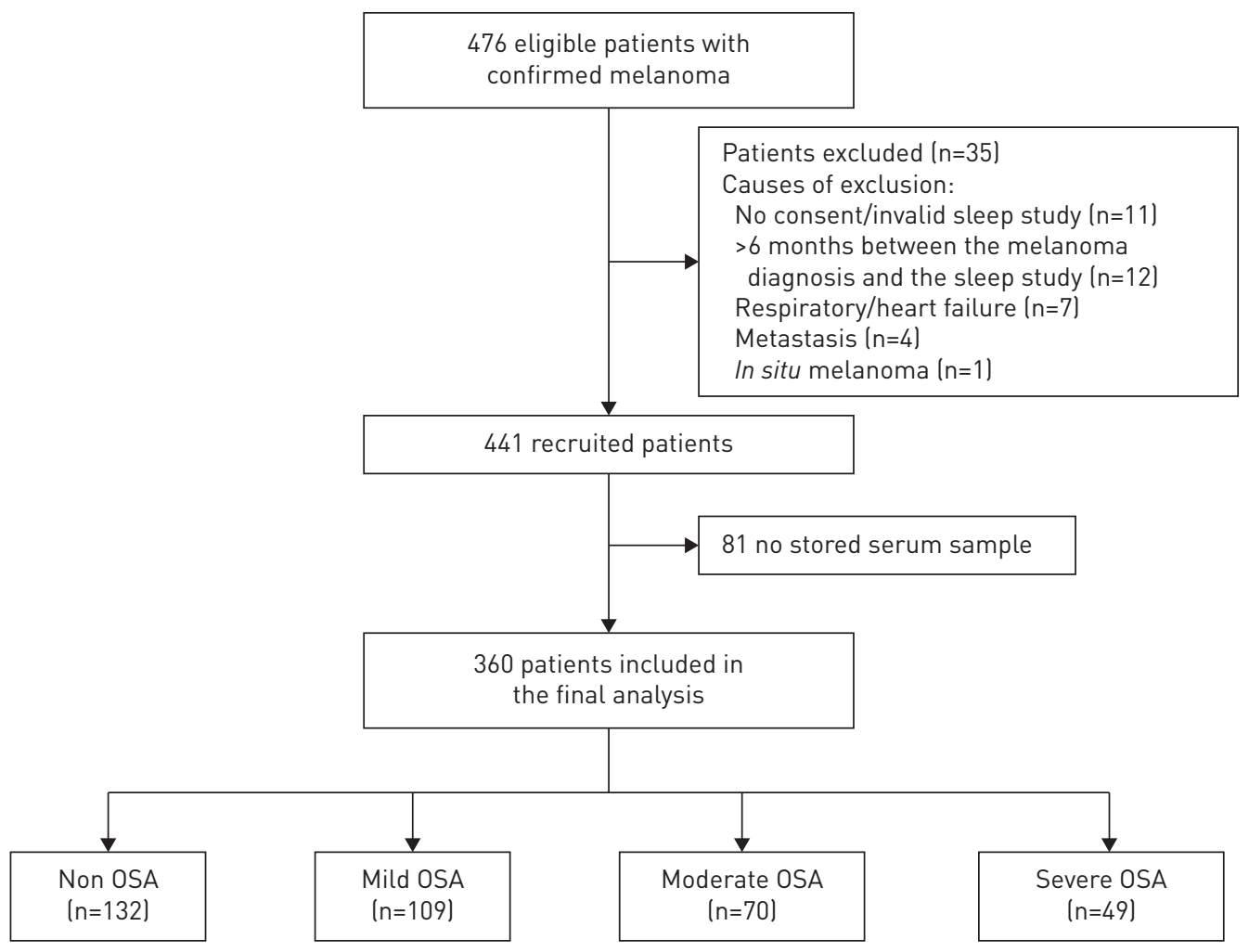

FIGURE 1 Flow chart of the study. OSA: obstructive sleep apnoea. 
TABLE 1 General characteristics of the study subjects

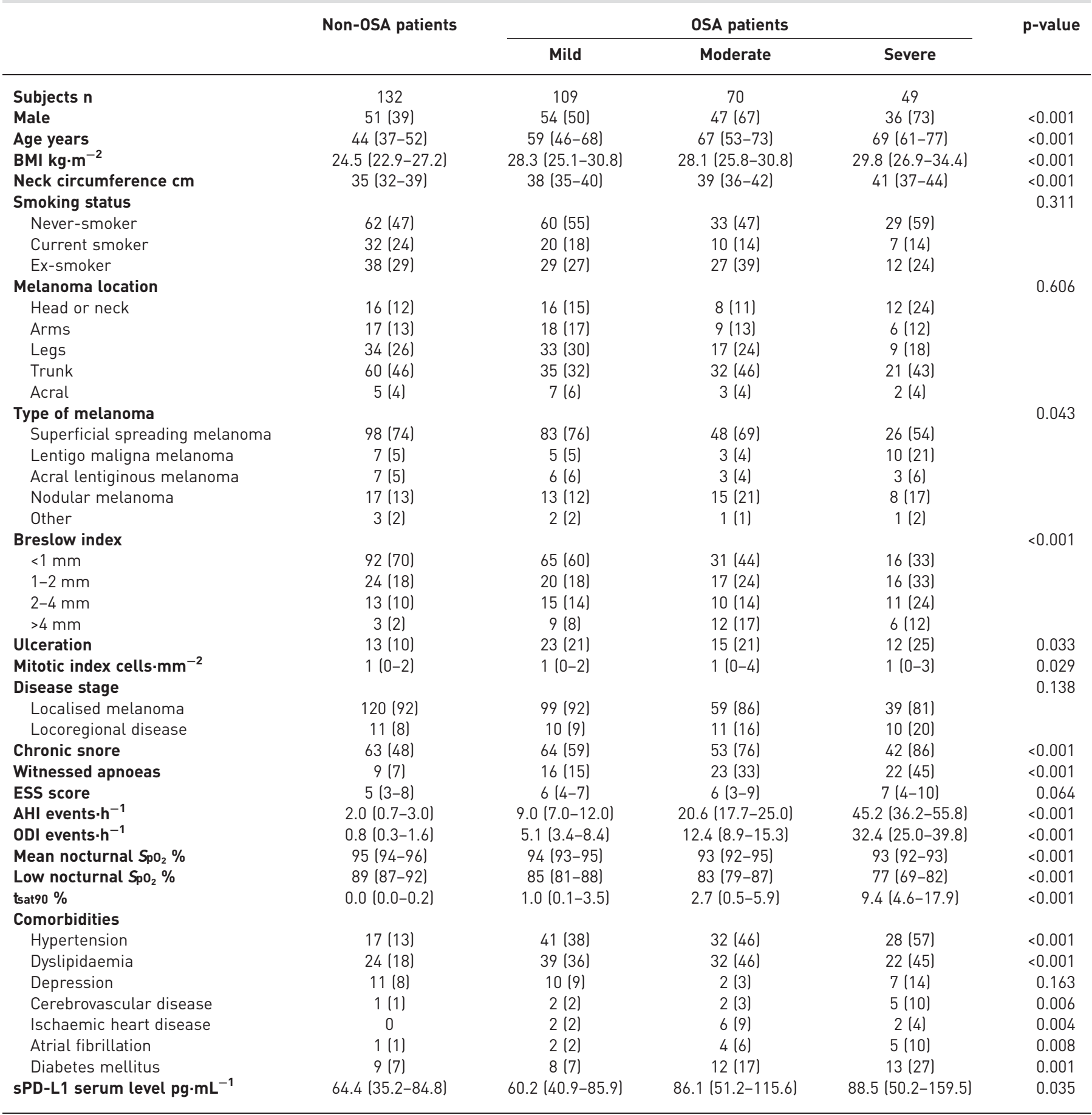

Data are presented as $\mathrm{n}(\%)$ or median (interquartile range), unless otherwise stated. OSA: obstructive sleep apnoea; BMI: body mass index; ESS: Epworth sleepiness score; $\mathrm{AHI}$ : apnoea-hypopnoea index; ODI: oxygen desaturation index; $\mathrm{SpO}_{2}$ : arterial oxygen saturation measured by pulse oximetry; tsat90: time spent at night with $\mathrm{SpO}_{2}<90 \%$; sPD-L1: soluble programmed cell death ligand-1.

$\mathrm{p}=0.013)$. Moreover, in OSA patients, we found a positive correlation between the Breslow index and sPD-L1 levels (figure 3), with significant differences between the degree of cutaneous melanoma thickness (figure 4). Specifically, sPD-L1 levels were $60.2(41.8-93.9) \mathrm{pg} \cdot \mathrm{mL}^{-1}$ in patients with cutaneous melanoma thickness $<1 \mathrm{~mm}$ and $92.1(76.2-134.9) \mathrm{pg} \cdot \mathrm{mL}^{-1}$ in patients with a thickness $>4 \mathrm{~mm}(\mathrm{p}=0.019)$. In addition, serum levels of sPD-L1 were higher in cutaneous melanoma patients with tumour ulceration when compared to those patients who did not have ulceration $\left(86.7(58.8-120.4) \mathrm{pg} \cdot \mathrm{mL}^{-1}\right.$ versus 71.5 
FIGURE 2 Box-and-whisker plots depicting the distribution of serum levels of soluble programmed cell death ligand-1 (sPD-L1) in cutaneous melanoma patients according to their apnoea-hypopnoea index. Data are presented as median (interquartile range), maximum and minimum values, and overall comparison was performed by the Kruskal-Wallis test. \#: p-values corresponding to the post hoc comparisons between groups adjusted by sex, age, body mass index and neck circumference. OSA: obstructive sleep apnoea.

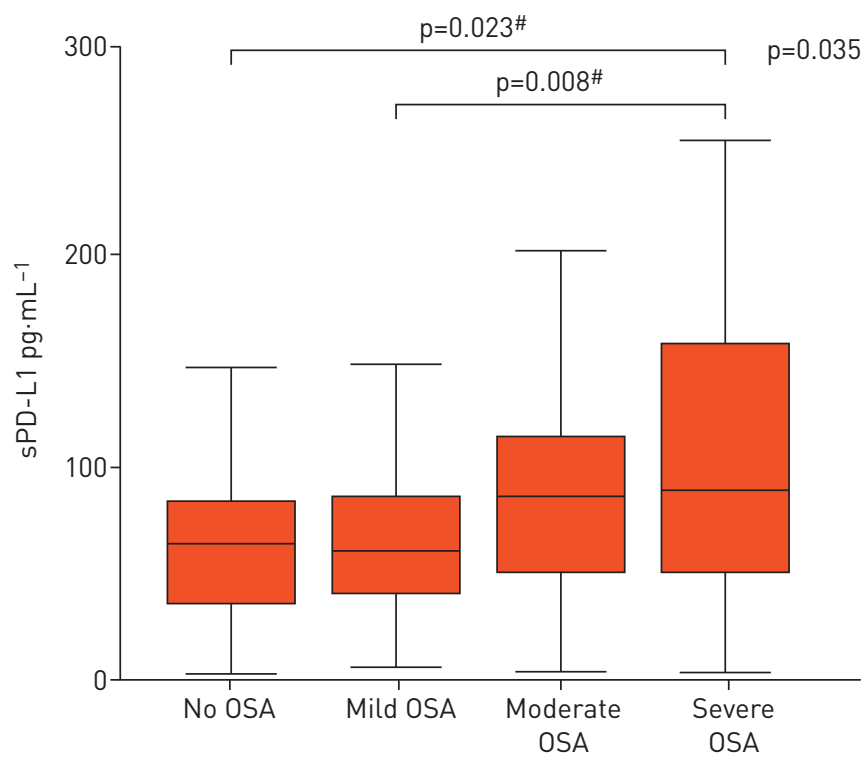

(41.4-99.4) $\left.\mathrm{pg} \cdot \mathrm{mL}^{-1} ; \mathrm{p}=0.035\right)$. Finally, a progressive increase in sPD-L1 levels was observed among the successive primary tumour categories (supplementary figure S1).

In contrast, no relationship was found between sPD-L1 and mitotic index (figure 3), and there were no differences in sPD-L1 levels between the Clark levels (70.2 (42.4-103.6) pg. $\mathrm{mL}^{-1}$ in patients at level 2 melanoma, $82.0(47.5-99.8) \mathrm{pg} \cdot \mathrm{mL}^{-1}$ in those at level 3, $75.0(37.8-113.8) \mathrm{pg} \cdot \mathrm{mL}^{-1}$ in those at level 4 and $101.7(76.3-145.0) \mathrm{pg} \cdot \mathrm{mL}^{-1}$ in those at level $\left.5 ; \mathrm{p}=0.136\right)$.

Of note, the levels of sPD-L1 of patients without OSA were not related to the Breslow or mitotic indices (supplementary figure S2), and they did not show differences depending on the presence of tumour ulceration, degrees of thickness, Clark levels or primary tumour categories (supplementary table S1).

SPD-L1 is a risk factor for sentinel lymph node metastasis in melanoma patients with OSA In 248 patients with melanoma and OSA, SLN biopsy was obtained and showed evidence of metastasis in $30(12.1 \%)$ cases. In a bivariate regression analysis, SLN metastasis was significantly associated with young age, mitotic index, Breslow index, tumour ulceration, Clark level and serum levels of sPD-L1 (table 3). SLN status was not associated with sex, BMI or any of the sleep study-derived parameters. However, a stepwise multivariate logistic regression analysis only retained four variables as independent risk factors for SLN metastasis, namely young age $(p=0.006)$, increased Breslow index $(p=0.025)$ and Clark level $(\mathrm{p}=0.026)$ and high sPD-L1 levels $(\mathrm{p}=0.008)$ (table 3$)$.

TABLE 2 Anthropometric and sleep parameters related to soluble programmed cell death ligand-1 serum levels in overall study subjects and obstructive sleep apnoea (OSA) patients

Overall patients

Spearman correlation
coefficient $\mathrm{r}(95 \% \mathrm{Cl})$

Age years

BMI $\mathrm{kg} \cdot \mathrm{m}^{-2}$

Neck circumference $\mathrm{cm}$

ESS

AHI events $\cdot h^{-1}$

ODI events $\cdot h^{-1}$

Mean nocturnal Sp02 \%

Low nocturnal Sp02 \%

tsat $90 \%$

$\begin{array}{cr}0.238(0.123-0.347) & <0.001 \\ 0.135(0.017-0.250) & 0.026 \\ 0.136(0.018-0.251) & 0.024 \\ 0.085(-0.034-0.201) & 0.162 \\ 0.206(0.090-0.317) & 0.001 \\ 0.233(0.118-0.342) & 0.001 \\ -0.232(-0.341--0.117) & <0.001 \\ -0.173(-0.286--0.056) & 0.004 \\ 0.239(0.124-0.348) & <0.001\end{array}$

\section{OSA patients}

Spearman correlation p-value
coefficient $r(95 \% \mathrm{CI})$

$0.224(0.083-0.356)$

$0.033(-0.111-0.176)$

$0.042(-0.103-0.185)$

$0.049(-0.096-0.192)$

$0.224(0.083-0.356)$

$0.258(0.119-0.387)$

$-0.230(-0.362--0.089)$

$-0.123(-0.262-0.021)$

$0.180(0.037-0.316)$
0.002

0.656

0.573

0.508

0.002

$<0.001$

0.002

0.095

0.014

BMI: body mass index; ESS: Epworth sleepiness score; AHI: apnoea-hypopnoea index; ODI: oxygen desaturation index; $\mathrm{ppO}_{2}$ : arterial oxygen saturation measured by pulse oximetry; tsat90: time spent at night with $\mathrm{SpO}_{2}<90 \%$. 

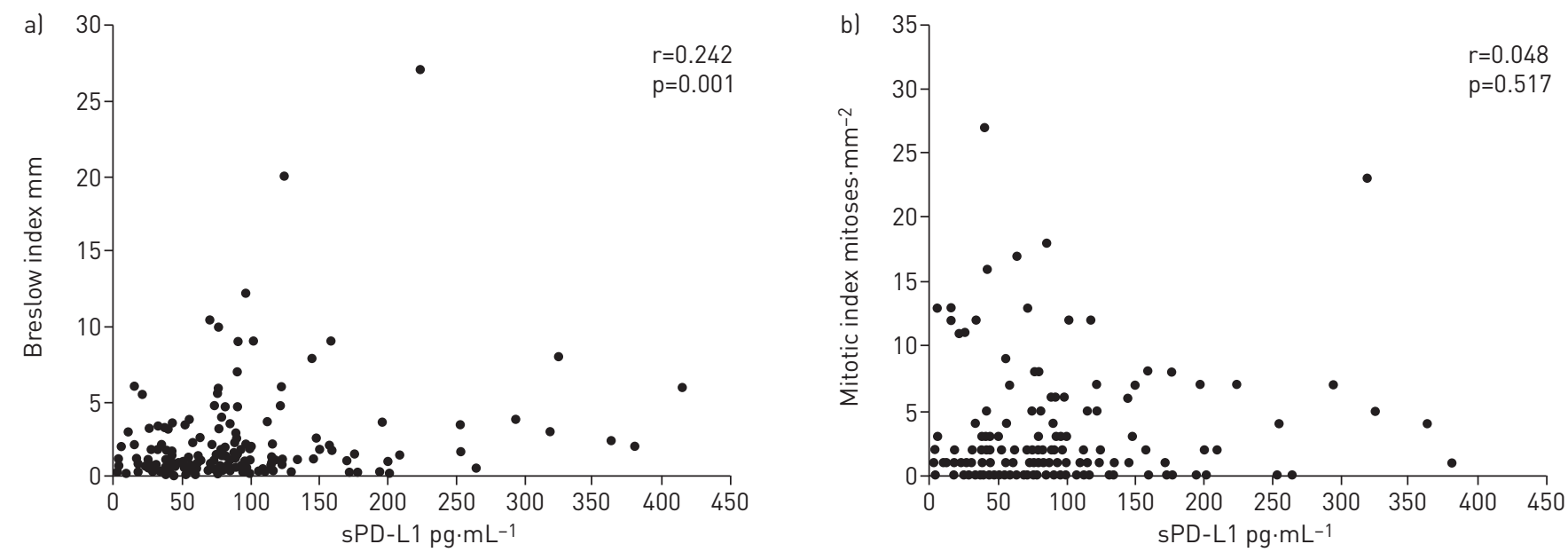

FIGURE 3 Relationship of the serum levels of soluble programmed cell death ligand-1 (sPD-L1) with a) the Breslow index and b) the mitotic index in patients with cutaneous melanoma and obstructive sleep apnoea.

For the individual prediction of positive SLN risk, an isolated sPD-L1 value had limited utility. The diagnostic accuracy of an optimal cut-off point (i.e. serum level $>74 \mathrm{pg} \cdot \mathrm{mL}^{-1}$ ) only reached a sensitivity of 75.0\% (95\% CI 53.0-89.4\%) and a specificity of 50.6\% (95\% CI 42.7-58.6\%). However, when sPD-L1 levels were incorporated into the classical prediction model based on conventional risk factors (age, Breslow depth, mitotic rate and Clark index), the AUC-ROC significantly increased ( $\mathrm{p}=0.024)$ from 0.837 (0.771-0.902) to $0.896(0.844-0.947)$ (figure 5). In turn, the incorporation of sPD-L1 to the classic risk model determined an IDI of $0.089(0.037-0.141 ; \mathrm{p}=0.001)$ and an NRI of $0.273(0.263-0.283 ; \mathrm{p}=0.036)$, indicating that the addition of sPD-L1 led to a net improvement in the classification of $27.3 \%$ of the cases.

\section{Discussion}

The present study shows that severe OSA is associated with increases in serum levels of sPD-L1 in patients with cutaneous melanoma. Moreover, we found that SPD-L1 levels in OSA patients are related to cutaneous melanoma aggressiveness and that they are an independent risk factor for the presence of SLN metastasis.

Similar to other molecules involved in immunoregulatory pathways, PD-L1 assumes two forms of expression, a membrane-bound ( $\mathrm{m}$ ) form and a soluble form. Usually, the soluble moiety is generated by proteolytic cleavage of the membrane-bound form of costimulatory proteins or by translation of alternative spliced mRNA [21,30]. Although other sources of sPD-L1 cannot be excluded, it seems probable that the PD-L1 expressed on the immune and tumour cell surface may provide the main source of the soluble PD-L1 form, since sPD-L1 is detectable in supernatants from $\mathrm{mPD}-\mathrm{L1}^{+}$cell lines, but not in those supernatants originating from mPD-L1 ${ }^{-}$cell lines [31]. Thus, the increases in sPD-L1 levels should be

FIGURE 4 Comparison of the serum levels of soluble programmed cell death ligand-1 (sPD-L1) according to the cutaneous melanoma thickness in obstructive sleep apnoea patients. Data are presented as median (interquartile range), maximum and minimum values. $p$-value corresponds to between-group comparison using the Kruskal-Wallis test.

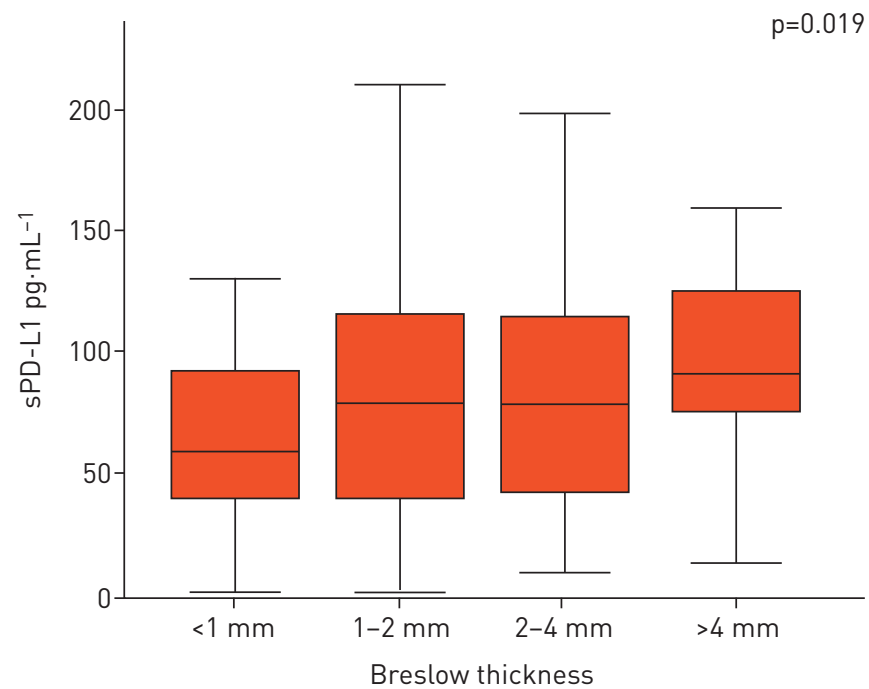


TABLE 3 Factors related to sentinel lymph node metastasis in patients with melanoma and obstructive sleep apnoea

\begin{tabular}{|c|c|c|c|c|}
\hline & \multicolumn{2}{|c|}{$\begin{array}{l}\text { Bivariate logistic } \\
\text { regression analysis }\end{array}$} & \multicolumn{2}{|c|}{$\begin{array}{c}\text { Stepwise multivariate } \\
\text { logistic regression analysis }\end{array}$} \\
\hline & OR $(95 \% \mathrm{CI})$ & p-value & OR $(95 \% \mathrm{CI})$ & p-value \\
\hline Male & $1.358(0.616-2.987)$ & 0.450 & & \\
\hline Age years & 0.988 (0.968-0.999) & 0.049 & 0.939 (0.897-0.982) & 0.006 \\
\hline $\mathrm{BMI} \mathrm{kg} \cdot \mathrm{m}^{-2}$ & $1.003(0.938-1.073)$ & 0.949 & & \\
\hline Current smoker & $0.938(0.395-2.228)$ & 0.884 & & \\
\hline ESS & 1.011 (0.909-1.123) & 0.845 & & \\
\hline $\mathrm{AHI}$ events $\cdot \mathrm{h}^{-1}$ & $1.009(0.989-1.031)$ & 0.376 & & \\
\hline ODI events: $h^{-1}$ & $1.013(0.996-1.029)$ & 0.130 & & \\
\hline Mean nocturnal $\mathrm{SpO}_{2} \%$ & $1.158(0.932-1.439)$ & 0.184 & & \\
\hline Low nocturnal $\mathrm{SpO}_{2} \%$ & 0.990 (0.957-1.025) & 0.581 & & \\
\hline tsat90 \% & $0.983(0.949-1.019)$ & 0.345 & & \\
\hline Mitotic index cells $\cdot \mathrm{mm}^{-2}$ & $1.155(1.074-1.241)$ & $<0.001$ & & \\
\hline Breslow index mm & 1.479 (1.270-1.723) & $<0.001$ & $1.371(1.041-1.805)$ & 0.025 \\
\hline Presence of ulceration & $5.909(2.646-13.196)$ & $<0.001$ & & \\
\hline Clark level & $4.961(2.690-9.152)$ & $<0.001$ & $3.286(1.155-9.346)$ & 0.026 \\
\hline Serum sPD-L1 pg:mL ${ }^{-1}$ & $1.009(1.004-1.014)$ & 0.001 & $1.009(1.002-1.016)$ & 0.008 \\
\hline
\end{tabular}

BMI: body mass index; ESS: Epworth sleepiness score; AHI: apnoea-hypopnoea index; ODI: oxygen desaturation index; $\mathrm{SpO}_{2}$ : arterial oxygen saturation measured by pulse oximetry; tsat90: time spent at night with $\mathrm{SpO}_{2}<90 \%$; sPD-L1: soluble programmed cell death ligand-1.

viewed as putative confirmation of the OSA contribution to upregulation of the PD-1/PD-L1 pathway, as recently described by our group [17].

Both in the whole cohort and in those patients who suffered from OSA, serum sPD-L1 levels were significantly correlated with age and with several polygraphic measures. The contribution of age is not novel, since it has been identified previously. Indeed, in normal subjects, serum levels of sPD-L1 increase with age, probably due to an increased activity of matrix metalloproteinases (MMPs), which would facilitate the cleavage of mPD-L1 [31]. With regards to sleep study-derived parameters, AHI emerged as the main determinant of sPD-L1 levels rather than some of the indices representing nocturnal hypoxaemia, especially considering that increases in PD-1/PD-L1 expression seem to be dependent on intermittent hypoxia and are mediated by hypoxia-inducible factor transcriptional activity [17]. It is possible that oxidative stress elicited by OSA may also favour increased sPD-L1 levels, promoting the

FIGURE 5 Receiver operating characteristic (ROC) curves for prediction of sentinel lymph node metastasis using the conventional risk factors lage, mitotic rate, Breslow index and Clark levels) alone or together with the new risk model, which includes serum levels of soluble programmed cell death ligand-1 (sPD-L1). AUC: area under the curve.

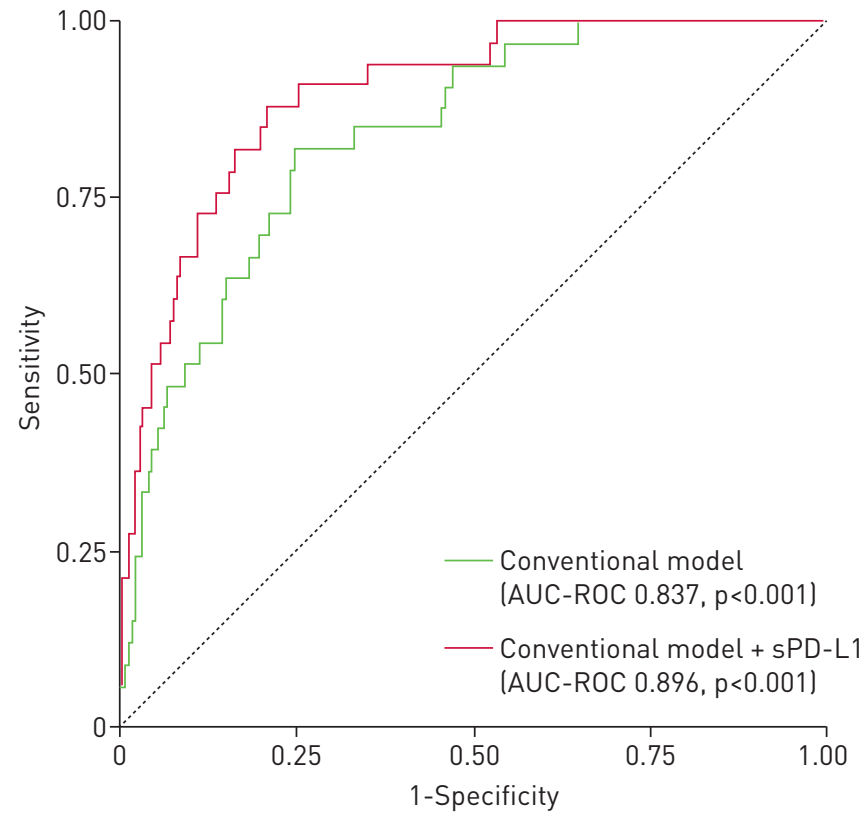


capacity of MMPs to cleave the extracellular fraction of MPD-L1. In fact, the link between oxidative stress and MMPs has been demonstrated in several experimental models [32], and it has been reported that the altered expression of certain MMPs and their tissue inhibitors in subjects with OSA [33, 34] correlates with several oxidative stress parameters [35].

In OSA patients with cutaneous melanoma, sPD-L1 levels were related to tumour aggressiveness and were higher in patients who present other poor prognostic indicators, such as the presence of tumour ulceration or higher tumour stage. It is particularly interesting to consider that sPD-L1 levels show a directly proportional relationship with an indicator of tumour aggressiveness, such as the Breslow index, while they do not reach statistical significance with an indicator of the intrinsic properties of the tumour cells, such as mitotic rate. This could reflect the importance of the interaction between the characteristics of the tumour cells and the immune system to determine the aggressiveness of the neoplastic disease. In any case, and according to the AJCC staging system, Breslow thickness and ulceration are considered, along with SLN involvement, the most important prognostic factors in melanoma patients, while mitotic rate is recognised only as a prognostic factor in the subgroup of thin melanomas [26].

Our findings concerning the relationship between sPD-L1 levels and melanoma aggressiveness agree with previous findings in other types of malignant tumours. Several studies have revealed that sPD-L1 levels are related to a higher aggressiveness and poorer prognosis in patients with nonsmall cell lung cancer, lymphoma, multiple myeloma and renal cell carcinoma [21, 36-38]. However, the mechanism by which sPD-L1 promotes tumour aggressiveness is more controversial. At present, it is still debatable whether sPD-L1 levels reflect only greater cellular expression of PD-L1 or if sPD-L1 can actually bind to PD-1, similarly to mPD-L1, thereby promoting immunosuppression [21]. In any case, both options represent an upregulation of the PD-1/PD-L1 pathway, which has recognised and well-established effects on tumour aggressiveness and progression. The activation of the PD-1/PD-L1 pathway can lead to tumour immune escape through several mechanisms, including the downregulation of the effector phase of T-cell immune responses by elevating the threshold of $\mathrm{T}$-cell activation, inhibiting $\mathrm{T}$-cell proliferation or by promoting T-cell apoptosis, as well as by enhancing immunosuppressive regulatory T-cell function $[36,39]$.

Surprisingly, in the patients of our study with melanoma but without OSA, no significant correlation emerged between sPD-L1 levels and tumour aggressiveness. This is particularly striking considering the aforementioned role of sPD-L1 in the promotion of immunity evasion and development and progression of several types of malignancies, including melanomas, as previously reported [40]. It is possible that the limited size of the group of patients with cutaneous melanoma but without OSA and the narrow aggressiveness spectrum may account for this apparent discrepancy. However, we should also point out that the presence or absence of OSA was not systematically explored in previous studies. Moreover, no association has been reported between tumour PD-L1 expression detected by immunohistochemical analysis and sPD-L1 levels in patients with some noncutaneous melanoma tumours. This suggests a major contribution of tumour microenvironment, including immune cells, to sPD-L1 generation [41]. Indeed, it has been demonstrated that PD-L1 is preferentially expressed on antigen-presenting cells, rather than on tumour cells, and plays an essential role in checkpoint blockade therapy [42].

In the management of patients with cutaneous melanoma, the importance of SLN biopsy lies in both the detection of occult nodal metastasis and as an indicator of overall treatment prognosis. However, SLN biopsy selection criteria are still under discussion. Conventionally, SLN biopsy is performed according to the risk estimated by the Breslow index, presence of tumour ulceration and Clark level [43]. However, the prediction capacity is not optimal, and other factors such as age or mitotic index have also been proposed as predictors of SLN positivity [26, 44].

Our study, which mainly involved patients with thin to intermediate thickness cutaneous melanoma reached an SLN positivity rate (12.1\%), which is similar to previous cohorts $[26,45]$, and highlights the potential usefulness of sPD-L1 levels in patients with cutaneous melanoma and OSA. Regarding classic risk factors, our findings are consistent with those previously established in the literature. In a recent cohort of 147 patients with stage I and II cutaneous melanoma, the Breslow index, Clark level, mitotic rate and presence of ulceration were identified as independent predictors of SLN metastasis [26]. However, the Breslow index is traditionally considered the most important predictor of SLN metastasis. In fact, in an analysis of 3460 patients with melanoma, multivariate analysis revealed young age and Breslow thickness as the only significant predictors, while mitotic rate and presence of ulceration were not retained in the model [45]. Although several studies found that ulceration or mitotic rate are associated with SLN metastasis [26, 43, 46-48], their role as independent predictors of SLN involvement has not been consistently established. Our findings suggest that increased sPD-L1 levels might also be a risk factor for SLN involvement in patients with cutaneous melanoma and OSA, which would confer them utility as a potential biomarker in this group of patients. 
However, the prediction capacity solely based on sPD-L1 levels is limited, such that use of sPD-L1 levels as a single risk factor is discouraged. However, the results of the present study show that incorporation of sPD-L1 into the classical risk models, together with the Breslow index, mitotic rate and presence of ulceration increases the predicting capacity of SLN metastasis in patients with cutaneous melanoma and OSA.

The main strengths of this study are its prospective nature, multicentre character and the relatively large cohort size, as well as the homogenisation and rigorous control of sleep studies performed by the Spanish Sleep Network. However, our study has several limitations that must be acknowledged. First, not all sleep variables potentially related to cancer, such as sleep fragmentation or even sleep habits or shift work, were documented and accounted for in our analyses. Second, there was some temporal delay between the skin biopsy and the sleep study and the collection of serum samples. Although we tried to minimise this time interval (which was not different between the study groups and never exceeded 5 months) and no changes in patient weight or any other potential confounding factors were identified, we cannot rule out that such factors were void of any influence in our results. Third, no biopsy tissues were available to determine PD-1 expression on the cutaneous melanoma tumour cells. Fourth, our results only refer to patients with cutaneous melanoma, such that the relationship between sPD-L1 levels and the aggressiveness and invasiveness of other cancer types in patients with OSA should be extrapolated cautiously. Fifth, our study does not provide any information on the effect of treatment of OSA on SPD-L1 levels or their effect on melanoma aggressiveness, and therefore no therapeutic recommendations beyond those in place for OSA criteria can be formulated.

In summary, the present study shows that melanoma patients with severe OSA have increased serum levels of soluble PD-L1. The addition of a simple assay, such the one required for determination of this parameter in patients with cutaneous melanoma and OSA, may provide a potential simple biomarker of aggressiveness and invasiveness in these patients. However, caution should be exercised and pathogenic inferences not made from our results, because although sPD-L1 seems to be a marker of aggressiveness, our data do not show that elevated SPD-L1 levels are necessarily the cause of aggressiveness. Although it is attractive to speculate that high SPD-L1 levels might reflect the presence of greater underlying degrees of immunosuppression due to upregulation of the PD-1/PD-L1 pathway, our data do not allow confirm this hypothesis. Both melanoma aggressiveness and OSA could raise PD-L1 levels, but the higher levels in OSA may not necessarily worsen melanomas.

Conflict of interest: None declared.

Support statement: This study was supported by grants from Fondo de Investigación Sanitaria (FIS) and Fondos FEDER PI13/01512 and PI16/00201 to F. García-Río and PI14/01234 and PIE15/00065 to E. López-Collazo. M.Á. Martínez-García is supported by the Spanish Ministry of Economy and Competitiveness - Instituto de Salud Carlos III (FIS 2016 / 01772) and cofinanced by the European Development Regional Fund; A way to achieve Europe (ERDF). Funding information for this article has been deposited with the Crossref Funder Registry.

\section{References}

1 Heinzer R, Vat S, Marques-Vidal P, et al. Prevalence of sleep-disordered breathing in the general population: the HypnoLaus study. Lancet Respir Med 2015; 3: 310-318.

2 Lévy P, Kohler M, McNicholas WT, et al. Obstructive sleep apnoea syndrome. Nat Rev Dis Primers 2015; 1: 15015.

3 Marin JM, Carrizo SJ, Vicente E, et al. Long-term cardiovascular outcomes in men with obstructive sleep apnoea-hypopnoea with or without treatment with continuous positive airway pressure: an observational study. Lancet 2005; 365: 1046-1053.

4 Sánchez-de-la-Torre M, Campos-Rodriguez F, Barbé F. Obstructive sleep apnoea and cardiovascular disease. Lancet Respir Med 2013; 1: 61-72.

5 Nieto FJ, Peppard PE, Young T, et al. Sleep-disordered breathing and cancer mortality: results from the Wisconsin Sleep Cohort Study. Am J Respir Crit Care Med 2012; 186: 190-194.

6 Campos-Rodriguez F, Martinez-Garcia MA, Martinez M, et al. Association between obstructive sleep apnea and cancer incidence in a large multicenter Spanish cohort. Am J Respir Crit Care Med 2013; 187: 99-105.

7 Martínez-García MA, Campos-Rodriguez F, Durán-Cantolla J, et al. Obstructive sleep apnea is associated with cancer mortality in younger patients. Sleep Med 2014; 15: 742-748.

8 Martínez-Garcia MA, Martorell-Calatayud A, Nagore E, et al. Association between sleep disordered breathing and aggressiveness markers of malignant cutaneous melanoma. Eur Respir J 2014; 43: 1661-1668.

9 Almendros I, Montserrat JM, Torres M, et al. Intermittent hypoxia increases melanoma metastasis to the lung in a mouse model of sleep apnea. Respir Physiol Neurobiol 2013; 186: 303-307.

10 Glazer AM, Farberg AS, Winkelmann RR, et al. Analysis of trends in geographic distribution and density of US dermatologists. JAMA Dermatol 2017; 153: 322-325.

11 Noguti J, Andersen ML, Cirelli C, et al. Oxidative stress, cancer, and sleep deprivation: is there a logical link in this association? Sleep Breath 2013; 17: 905-910.

12 Martínez-García MA, Campos-Rodríguez F, Farre R. Sleep apnoea and cancer: current insights and future perspectives. Eur Respir J 2013; 40: 1315-1317. 
13 Almendros I, Wang Y, Becker L, et al. Intermittent hypoxia-induced changes in tumor-associated macrophages and tumor malignancy in a mouse model of sleep apnea. Am J Respir Crit Care Med 2014; 189: 593-601.

14 Hakim F, Wang Y, Zhang SX, et al. Fragmented sleep accelerates tumor growth and progression through recruitment of tumor-associated macrophages and TLR4 signaling. Cancer Res 2014; 74: 1329-1337.

15 Akbarpour M, Khalyfa A, Qiao Z, et al. Altered CD8 ${ }^{+}$T-cell lymphocyte function and TC1 cell stemness contribute to enhanced malignant tumor properties in murine models of sleep apnea. Sleep 2017; 40: zsw040.

16 Hernández-Jiménez E, Cubillos-Zapata C, Toledano V, et al. Monocytes inhibit NK activity via TGF- $\beta$ in patients with obstructive sleep apnoea. Eur Respir J 2017; 49: 1602456.

17 Cubillos-Zapata C, Avendaño-Ortiz J, Hernandez-Jimenez E, et al. Hypoxia-induced PD-L1/PD-1 crosstalk impairs T-cell function in sleep apnoea. Eur Respir J 2017; 50: 1700833.

18 Mahoney KM, Freeman GJ, McDermott DF. The next immune-checkpoint inhibitors: PD-1/PD-L1 blockade in melanoma. Clin Ther 2015; 37: 764-782.

19 Nagato T, Ohkuri T, Ohara K, et al. Programmed death-ligand 1 and its soluble form are highly expressed in nasal natural killer/T-cell lymphoma: a potential rationale for immunotherapy. Cancer Immunol Immunother 2017; 66: $877-890$.

20 Zhou J, Mahoney KM, Giobbie-Hurder A, et al. Soluble PD-L1 as a biomarker in malignant melanoma treated with checkpoint blockade. Cancer Immunol Res 2017; 5: 480-492.

21 Zhu X, Lang J. Soluble PD-1 and PD-L1: predictive and prognostic significance in cancer. Oncotarget 2017; 8: 97671-97682.

22 Morton DL, Thompson JF, Cochran AJ, et al. Final trial report of sentinel-node biopsy versus nodal observation in melanoma. N Engl J Med 2014; 370: 599-609.

23 Santamaria-Martos F, Benítez I, Girón C, et al. Biomarkers of carcinogenesis and tumour growth in patients with cutaneous melanoma and obstructive sleep apnoea. Eur Respir J 2018; 51: 1701885.

24 Martínez-García MA, Riveiro-Falkenbach E, Rodríguez-Peralto JL, et al. A prospective multicenter cohort study of cutaneous melanoma: clinical staging and potential associations with HIF-1 $\alpha$ and VEGF expressions. Melanoma Res 2017; 27: 558-564.

25 Gershenwald JE, Scolyer RA, Hess KR, et al. Melanoma staging: evidence-based changes in the American Joint Committee on Cancer eighth edition cancer staging manual. CA Cancer J Clin 2017; 67: 472-492.

26 Jokic S, Markovic I, Bukumiric Z, et al. Predictors of sentinel lymph node status of cutaneous melanoma in Serbian patients. J BUON 2018; 23: 468-474.

27 Lloberes P, Durán-Cantolla J, Martínez-García MÁ, et al. Diagnosis and treatment of sleep apnea-hypopnea syndrome. Spanish Society of Pulmonology and Thoracic Surgery. Arch Bronconeumol 2011; 47: 143-156.

28 DeLong ER, DeLong DM, Clarke-Pearson DL. Comparing the areas under two or more correlated receiver operating characteristic curves: a nonparametric approach. Biometrics 1988; 44: 837-845.

29 Uno H, Tian L, Cai T, et al. A unified inference procedure for a class of measures to assess improvement in risk prediction systems with survival data. Stat Med 2013; 32: 2430-2442.

30 Zhang G, Hou J, Shi J, et al. Soluble CD276 (B7-H3) is released from monocytes, dendritic cells and activated T cells and is detectable in normal human serum. Immunology 2008; 123: 538-546.

31 Chen Y, Wang Q, Shi B, et al. Development of a sandwich ELISA for evaluating soluble PD-L1 (CD274) in human sera of different ages as well as supernatants of PD-L1+ cell lines. Cytokine 2011; 56: 231-238.

32 Viappiani S, Nicolescu AC, Holt A, et al. Activation and modulation of $72 \mathrm{kDa}$ matrix metalloproteinase-2 by peroxynitrite and glutathione. Biochem Pharmacol 2009; 77: 826-834.

33 Chuang LP, Chen NH, Lin SW, et al. Increased matrix metalloproteinases-9 after sleep in plasma and in monocytes of obstructive sleep apnea patients. Life Sci 2013; 193: 220-225.

34 Tazaki T, Minoguchi K, Yokoe T, et al. Increased levels and activity of matrix metalloproteinase- 9 in obstructive sleep apnea syndrome. Am J Respir Crit Care Med 2004; 170: 1354-1359.

35 Hopps E, Lo Presti R, Montana M, et al. Analysis of the correlations between oxidative stress, gelatinases and their tissue inhibitors in the human subjects with obstructive sleep apnea syndrome. J Physiol Pharmacol 2015; 66: 803-810.

36 Zhang J, Gao J, Li Y, et al. Circulating PD-L1 in NSCLC patients and the correlation between the level of PD-L1 expression and the clinical characteristics. Thorac Cancer 2015; 6: 534-538.

37 Wang L, Wang $\mathrm{H}$, Chen $\mathrm{H}$, et al. Serum levels of soluble programmed death ligand 1 predict treatment response and progression free survival in multiple myeloma. Oncotarget 2015; 6: 41228-41236.

38 Frigola X, Inman BA, Lohse CM, et al. Identification of a soluble form of B7-H1 that retains immunosuppressive activity and is associated with aggressive renal cell carcinoma. Clin Cancer Res 2011; 17: 1915-1923.

39 Palucka AK, Coussens LM. The basis of oncoimmunology. Cell 2016; 164: 1233-1247.

40 Tsai KK, Zarzoso I, Daud AI. PD-1 and PD-L1 antibodies for melanoma. Hum Vaccin Immunother 2014; 10: 3111-3116.

41 Ruf M, Moch H, Schraml P. PD-L1 expression is regulated by hypoxia inducible factor in clear cell renal cell carcinoma. Int J Cancer 2016; 139: 396-403.

42 Tang H, Liang Y, Anders RA, et al. PD-L1 on host cells is essential for PD-L1 blockade-mediated tumor regression. J Clin Invest 2018; 128: 580-588.

43 Oláh J, Gyulai R, Korom I, et al. Tumour regression predicts higher risk of sentinel node involvement in thin cutaneous melanomas. Br J Dermatol 2003; 149: 662-663.

44 Kesmodel SB, Karakousis GC, Botbyl JD, et al. Mitotic rate as a predictor of sentinel lymph node positivity in patients with thin melanomas. Ann Surg Oncol 2005; 12: 449-458.

45 Chang JM, Kosiorek HE, Dueck AC, et al. Stratifying SLN incidence in intermediate thickness melanoma patients. Am J Surg 2018; 215: 699-706.

46 Azzola MF, Shaw HM, Thompson JF, et al. Tumor mitotic rate is a more powerful prognostic indicator than ulceration in patients with primary cutaneous melanoma: an analysis of 3661 patients from a single center. Cancer 2003; 97: 1488-1498.

47 Sartore L, Papanikolaou GE, Biancari F, et al. Prognostic factors of cutaneous melanoma in relation to metastasis at the sentinel lymph node: a case-controlled study. Int J Surg 2008; 6: 205-209.

48 Faries MB, Thompson JF, Cochran AJ, et al. Completion dissection or observation for sentinel-node metastasis in melanoma. N Engl J Med 2017; 376: 2211-2222. 